\title{
Progression of Pseudomyxoma Peritonei after Combined Modality Treatment: Management and Outcome
}

\author{
Robert M. Smeenk, MD, ${ }^{1}$ Vic J. Verwaal, MD, PhD,${ }^{1}$ Ninja Antonini, $\mathrm{MSc},{ }^{2}$ and \\ Frans A. N. Zoetmulder, $\mathrm{MD}, \mathrm{PhD}^{1}$
}

${ }^{1}$ Department of Surgical Oncology, The Netherlands Cancer Institute, Amsterdam, The Netherlands ${ }^{2}$ Department of Biometrics, The Netherlands Cancer Institute, Amsterdam, The Netherlands

Background: Cytoreductive surgery (CRS) with hyperthermic intraperitoneal chemotherapy (HIPEC) is a treatment strategy for pseudomyxoma peritonei (PMP) with curative intent. The aim of this study was to determine the patterns of failure in patients who underwent such a procedure and to evaluate management and outcome of progressive disease.

Methods: After exclusion of patients with overt malignancy, progression was studied in 96 PMP patients treated primarily by CRS with HIPEC. Location, pathology, management and outcome were recorded.

Results: Median follow-up was 51.5 months (0.1-99.5). Median progression free survival (PFS) was 28.2 months (95\% CI 18.3->). Progressive disease was mainly located sub hepatic $(38 \%)$ or in multiple regions $(36 \%)$. Pathological dedifferentiation was observed in 8 patients (20\%). The choice of treatment depended on pathology, extent of disease and PFS. Seventeen patients were treated for progression by second CRS with $(n=8)$ or without HIPEC $(n=10)$. The 3-years overall survival (OS) probability after this treatment was $100 \%$ and $53.3 \%(95 \%$ CI 28.2-100\%), respectively. Fifteen patients with (slow) progression were observed. Threeyears OS probability of these patients was $66.0 \%$ (95\% CI 43.4-100\%). All patients treated for progression by systemic chemotherapy only $(n=6)$ had died of disease after a median follow up of 14.8 (9.8-33.6) months. A longer PFS after primary treatment was associated with longer OS after progression $(P=0.04)$.

Conclusions: Progressive PMP after primary CRS with HIPEC is probably the result of technical failure and/or tumor biology. Management of progressive PMP can be valuable for selected patients and should depend primarily on the PFS.

Key Words: Pseudomyxoma peritonei-Cytoreduction-HIPEC - Progression-Management Outcome.

Pseudomyxoma peritonei (PMP) is a rare disease characterized by peritoneal deposits of adenomucinous tumor cells producing a progressive amount of intraperitoneal mucinous ascites. These intraperitoneal disseminated tumor cells usually originate from a ruptured appendiceal neoplasm. The process of

Received May 18, 2006; accepted May 18, 2006; published online November 11, 2006.

Address correspondence and reprint requests to: Vic J. Verwaal, MD, PhD; E-mail: v.verwaal@nki.nl

Published by Springer Science+Business Media, Inc. (c) 2006 The Society of Surgical Oncology, Inc. dissemination throughout the peritoneal cavity is described as the redistribution phenomenon. ${ }^{1}$

Aggressive cytoreductive surgery (CRS) with hyperthermic intraperitoneal chemotherapy (HIPEC) is a treatment strategy for PMP with promising survival results. ${ }^{2-5}$ The peritonectomy procedures are a useful tool to optimize resection of macroscopic peritoneal implants. HIPEC seems to reduce the recurrence or progression rate significantly by the cytotoxic effect on (microscopic) tumor residue, especially in patients with low-grade pathology and complete cytoreduction. ${ }^{3,5-7}$ Recurrent or progressive disease after this combined modality treatment is 
however, still a problem. Treatment approaches for recurrent or progressive PMP are not well described, although surgery seems to be the most common option. ${ }^{8-10}$ At The Netherlands Cancer Institute, the standard primary treatment of PMP patients consists of aggressive CRS by peritonectomy procedures in combination with intraoperative HIPEC with Mitomycin C. Aim of this study is to determine the patterns of failure after this combined modality treatment and to evaluate the management and outcome of progressive PMP.

\section{METHODS}

\section{Primary Treatment}

A prospective database of PMP patients treated at The Netherlands Cancer Institute has been maintained from 1996 onwards. Patients treated in the period 1996-2004 were retrospectively analyzed. All patients were primarily treated with aggressive CRS (peritonectomy procedures) with intraoperative HIPEC, a procedure described in detail elsewhere. ${ }^{11}$ The result (completeness) of cytoreduction was recorded at the end of surgery: no residual macroscopic tumor (R1 cytoreduction), residual macroscopic tumor $\leq 2.5 \mathrm{~mm}$ in any region (R2a cytoreduction) and tumor deposits $>2.5 \mathrm{~mm}$ in any region ( $\mathrm{R} 2 \mathrm{~b}$ cytoreduction).

\section{Pathological Subtypes}

For analysis of this study, PMP was categorized into three pathological subtypes: disseminated peritoneal adenomucinosis (DPAM), peritoneal mucinous carcinomatosis (PMCA) and an intermediate subtype (PMCA-I). ${ }^{12}$ DPAM pathology shows characteristics of benign tumor containing no or very few epithelial cells with no or little atypia. PMCA pathology includes tumor containing epithelial cells with malignant phenotype. PMCA-I is an intermediate subtype with mainly features of DPAM but focally characteristics of PMCA. Patients with PMCA pathology at primary presentation were excluded from this analysis as we feel that PMCA should be categorized as peritoneal carcinomatosis and not as PMP.

\section{Follow-Up}

Follow-up consisted of physical examination and assessment of tumor markers CEA and CA $19.9 \mathrm{ev-}$ ery 3 months in the first year. In the following years patients were seen every 6 months. Abdominal com- puted tomography (CT) scan was performed 3 months after the primary treatment and after that at every 6 months follow-up visit or when recurrence or progression was suspected.

Policy towards recurrent or progressive disease in the period 1996-2004 was based on the following principles with important selection criteria consisting of the extent of disease (visualized on follow-up CT scans), pathology and time to recurrent or progressive disease. Patients with limited ( $<3$ regions) and resectable disease, both DPAM and PMCA-I subtype, were treated with local resection only. Patients with limited slowly progressive disease, especially DPAM but also PMCA-I subtype, were observed without therapy. Patients with early-developed diffuse disease of initial PMCA-I subtype or initial DPAM with signs of dedifferentiation at progression or with signs of extraperitoneal disease were treated with 5-FU based systemic chemotherapy. Patients with late-developed diffuse disease of benign subtype (DPAM) were treated with second CRS plus HIPEC.

\section{Data Analysis and Statistical Methods}

Both recurrent PMP after an R1 cytoreduction and progressive disease after an R2 cytoreduction was defined as progressive PMP for analysis in this study. The outcome of primary treatment was analyzed in terms of progression free survival (PFS), calculated from date of primary treatment to date of first progression or date of last follow-up. Progressive PMP was studied with regard to its location, pathology, management and outcome. The location of progressive PMP was identified in five abdominal regions (pelvis, small or large bowel and mesentery/omentum, sub hepatic region and right and left sub phrenic region), on surgical wound surfaces or extraperitoneal. When the site of progression was not obvious, it was categorized as not clearly defined. Pathological dedifferentiation was defined as a difference in pathology between PMP at primary treatment and progressive PMP from DPAM towards PMCA-I or PMCA. Patients with missing data were excluded.

Treatment for the first progression was recorded. Time to second progression was calculated from date of first progression to date of second progression or date of last follow-up. The outcome of management for progressive PMP was analyzed in terms of PFS probability and overall survival (OS) probability, calculated from date of first progression to second progression or date of last follow-up. Patients with missing data were excluded. Survival (probability) was analyzed according to the Kaplan Meier esti- 
TABLE 1. Result of initial cytoreduction associated with pathology at time of diagnosis and location of disease in patients with progressive PMP after primary CRS and HIPEC

\begin{tabular}{|c|c|c|c|c|}
\hline & \multirow[b]{2}{*}{$\mathrm{n}(\%)$} & \multicolumn{3}{|c|}{ Result of initial cytoreduction ${ }^{a}$} \\
\hline & & $\mathrm{R} 1$ & $\mathrm{R} 2 \mathrm{a}$ & $\mathrm{R} 2 \mathrm{~b}$ \\
\hline Patients & 91 & 29 & 52 & 10 \\
\hline DPAM & 64 & 21 & 36 & 7 \\
\hline PMCA-I & 27 & 8 & 16 & 3 \\
\hline Progression (\%) & $39(43)$ & $4(14)$ & $28(54)$ & $7(70)$ \\
\hline DPĀM & $25(39)$ & $2(10)$ & $18(50)$ & $5(71)$ \\
\hline PMCA-I & $14(52)$ & $2(25)$ & $10(63)$ & $2(67)$ \\
\hline \multicolumn{5}{|l|}{ Location } \\
\hline Sub hepatic & $15(38)$ & 2 & 11 & 2 \\
\hline Pelvis & $3(8)$ & - & 2 & 1 \\
\hline Bowel & $2(5)$ & - & 2 & - \\
\hline Abdominal scar & $1(3)$ & - & 1 & - \\
\hline Multiple sites & $14(36)$ & 2 & 9 & 3 \\
\hline Not clearly defined & $4(10)$ & - & 3 & 1 \\
\hline
\end{tabular}

PMP, pseudomyxoma peritonei, CRS, cytoreductive surgery, HIPEC, hyperthermic intraperitoneal chemotherapy, R1, no macroscopic tumor residue, $\mathrm{R} 2 \mathrm{a}$, macroscopic residue $\leq 2.5 \mathrm{~mm}$ in any region, $\mathrm{R} 2 \mathrm{~b}$, macroscopic residue $>2.5 \mathrm{~mm}$ in any region, DPAM, disseminated peritoneal adenomucinosis, PMCA-I, intermediate pathological subtype.

${ }^{a}$ Result of initial cytoreduction could be determined in $91 / 96$ patients.

mation method. A Cox's proportional hazards regression model was used to analyze the effect of pathological subtype and result of cytoreduction on PFS. Significance was defined as a $P$-value $<0.05$.

\section{RESULTS}

\section{Primary Treatment}

Ninety-six patients with PMP, 31 males and 65 females, were primarily treated with CRS and intraoperative HIPEC. The median age was 57 years (3077). Result of cytoreduction could be determined in 91 of 96 patients. A macroscopic complete cytoreduction (R1) was reached in $32 \%$ of patients. An R2a cytoreduction was performed in $57 \%$ of patients and an R2b cytoreduction in 11\%. Pathological subtype at primary treatment was DPAM in 66 patients and PMCA-I in 30 patients. After a median follow-up of 51.5 months (0.1-99.5) months progression was observed in $41 \%(39 / 96)$ of patients with a median PFS of 28.2 months (95\% CI > 18.3).

\section{Presentation of Progressive Disease}

Progression was diagnosed in case of a marked rise of tumor marker(s) and evidence of relapse on abdominal CT scan, or during laparotomy for any cause. Progression of PMP was related to the result of initial cytoreduction and initial pathology, as shown in Table 1 and Fig. 1. Table 1 shows the locations of progressive disease. Progressive disease on surgical wound surfaces $(\mathrm{n}=11)$ was confined to stomach/ bowel $(\mathrm{n}=6)$, abdominal wall/scar $(\mathrm{n}=3)$, colostomy $(\mathrm{n}=1)$, and vagina-top $(\mathrm{n}=1)$. Extraperitoneal disease was observed in the pleural cavity $(n=4)$ and retroperitoneal in the ureter $(n=1)$.

Pathology at progression was categorized as DPAM or PMCA-I in 25 and 14 patients, respectively. A trend was observed in the association between progression and pathological subtype (Table 1; Fig. 1a), but significance was not reached (Chisquare, $P>0.05)$. Dedifferentiation was observed in $8 / 39$ patients $(20 \%)$. In four patients dedifferentiation was diagnosed at biopsy or obduction. In another four patients early aggressive progression $(n=3)$ or distant metastasis $(\mathrm{n}=1)$ strongly suggested dedifferentiation compared to the original DPAM histology. In four of these eight patients progressive disease was located in multiple regions.

\section{Management and Outcome of Progressive Disease}

Table 2 shows the management and outcome of progressive disease and the association of the choice of treatment with pathological subtype, result of initial cytoreduction and PFS. The median interval between diagnosis of first progression and surgical treatment was 3.4 months (range 0.5-53.1). Figure 2 shows the impact of PFS (after initial CRS) on the OS probability (after the first progression) in patients with progressive disease.

The 3-year PFS probability and the 3-year OS probability after a second procedure of CRS and HIPEC $(\mathrm{n}=8)$ was $42.9 \%(95 \%$ CI $18.2-100 \%)$ and 

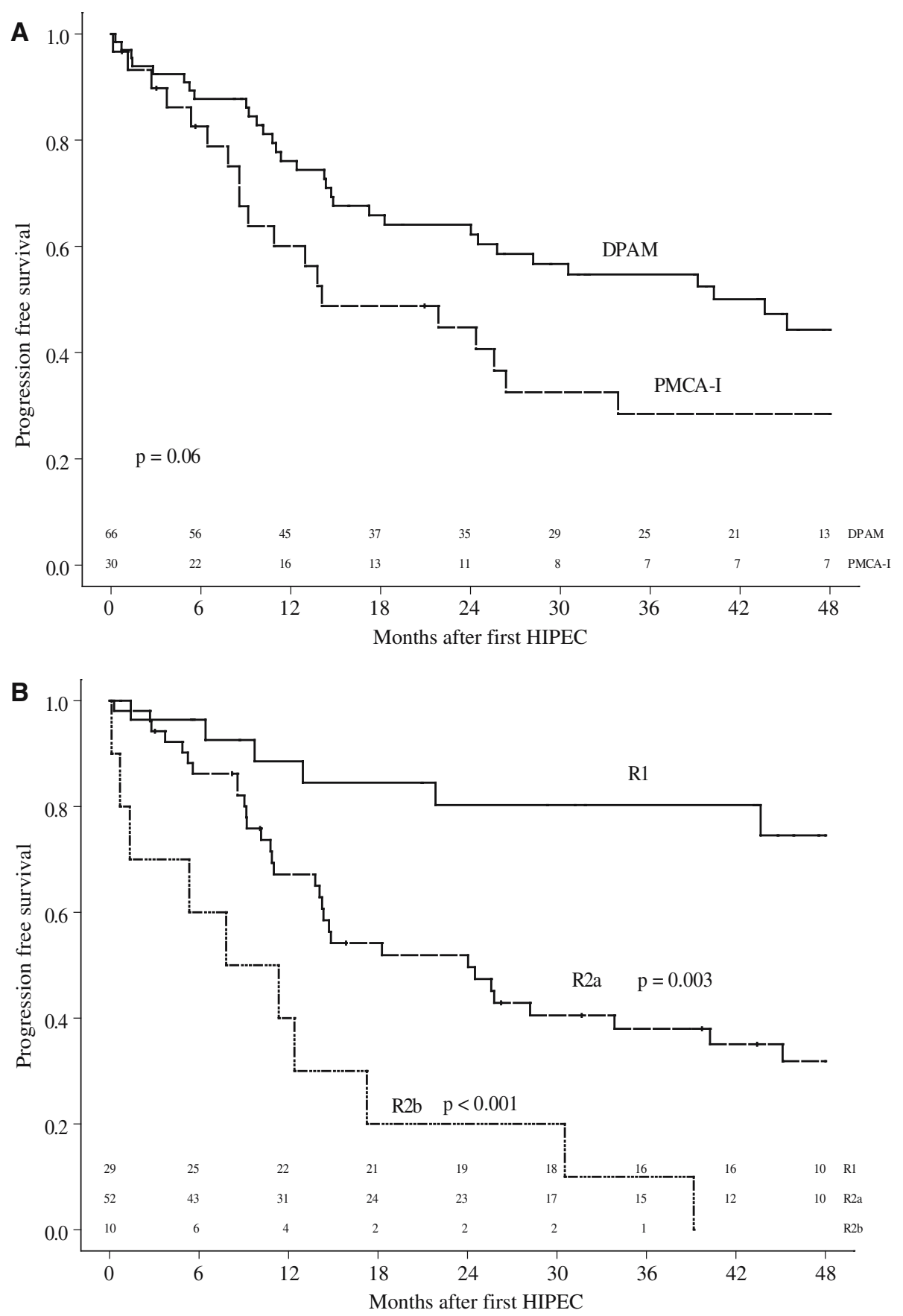

FIG. 1. a Progression free survival related to pathological subtype at time of diagnosis in PMP patients after primary CRS and HIPEC. PMP, pseudomyxoma peritonei, CRS, cytoreductive surgery, HIPEC, hyperthermic intraperitoneal chemotherapy, DPAM, disseminated peritoneal adenomucinosis, PMCA-I, intermediate pathological subtype. b Progression free survival related to result of initial CRS in PMP patients after primary CRS and HIPEC. PMP, pseudomyxoma peritonei, CRS, cytoreductive surgery, HIPEC, hyperthermic intraperitoneal chemotherapy, $\mathrm{R} 1$, no macroscopic tumor residue, $\mathrm{R} 2 \mathrm{a}$, macroscopic residue $\leq 2.5 \mathrm{~mm}$ in any region, $\mathrm{R} 2 \mathrm{~b}$, macroscopic residue $>2.5 \mathrm{~mm}$ in any region.

$100 \%$, respectively. In two of these patients systemic chemotherapy was given prior to the second procedure.

In case of a surgical intervention only $(\mathrm{n}=10) 3$ year PFS probability and 3-year OS probability was $22.2 \%$ (95\% CI $6.5-75.4 \%$ ) and $53.3 \%$ (95\% CI $28.2-100 \%$ ), respectively. In two of these patients, with DPAM subtype, a second procedure of CRS and HIPEC had been performed for a second pro- gression 3 years after the first. Both patients had no evidence of disease at end of follow-up. One patient, with local progression of DPAM in the abdominal scar, had been treated three times with a local resection. At end of follow-up, 47.9 months after the primary treatment there was no evidence of disease.

Fifteen patients with progression were observed and $42.9 \%$ of these patients (95\% CI $18.2-100 \%$ ) 
TABLE 2. Management and outcome of progressive PMP after primary CRS with HIPEC, associated with pathology at time of diagnosis, result of initial CRS and PFS after primary treatment

\begin{tabular}{|c|c|c|c|c|c|c|}
\hline Extent & $\begin{array}{l}\text { Treatment for } \\
\text { progression (n) }\end{array}$ & $\begin{array}{l}\text { Initial } \\
\text { pathology (n) }\end{array}$ & $\begin{array}{l}\text { Result of } \\
\text { initial CRS (n) }\end{array}$ & $\begin{array}{c}\text { Median PFS } \\
\text { after initial } \\
\text { CRS and HIPEC, } \\
\text { in months }(95 \% \mathrm{CI})\end{array}$ & $\begin{array}{l}\text { Median FU } \\
\text { after treatment } \\
\text { for progression, } \\
\text { in months (range) }\end{array}$ & Outcome (n) \\
\hline \multirow[t]{2}{*}{ Limited } & Surgery only (10) & $\begin{array}{l}\text { DPAM (4) } \\
\text { PMCA-I (6) }\end{array}$ & $\begin{array}{l}\text { R1 (1) } \\
\text { R2a (8) } \\
\text { R2b (1) }\end{array}$ & $21.7(14.4-33.6)$ & $18.1(2.3-49.6)$ & $\begin{array}{l}\text { NED (6) } \\
\text { AWD (1) } \\
\text { DOD (3) }\end{array}$ \\
\hline & Observation (15) & $\begin{array}{l}\text { DPAM (10) } \\
\text { PMCA-I (5) }\end{array}$ & $\begin{array}{l}\text { R1 (2) } \\
\text { R2a (8) } \\
\text { R2b (5) }\end{array}$ & $64.4(>15.8)$ & $16.8(3.4-70.3)$ & $\begin{array}{l}\text { AWD (11) } \\
\text { DOD (4) }\end{array}$ \\
\hline \multirow[t]{2}{*}{ Diffuse } & 2nd HIPEC (8) & DPAM (8) & $\begin{array}{l}\text { R1 (1) } \\
\text { R2a (7) }\end{array}$ & $34.1(13.8-68.3)$ & $19.3(8.8-56.6)$ & $\begin{array}{l}\text { NED (2) } \\
\text { AWD (2) } \\
\text { DOD (4) }\end{array}$ \\
\hline & Systemic chemo only (6) & $\begin{array}{l}\text { DPAM (3) } \\
\text { PMCA-I (3) }\end{array}$ & $\begin{array}{l}\text { R2a (5) } \\
\text { R2b (1) }\end{array}$ & $14.3(12.8-17.3)$ & $14.8(9.8-33.6)$ & DOD (6) \\
\hline
\end{tabular}

PMP, pseudomyxoma peritonei, CRS, cytoreductive surgery, HIPEC, hyperthermic intraperitoneal chemotherapy, PFS, progression free survival, CI, confidence interval, FU, follow-up, DPAM, disseminated peritoneal adenomucinosis, PMCA-I, intermediate pathological subtype, R1, no macroscopic tumor residue, $\mathrm{R} 2 \mathrm{a}$, macroscopic residue $\leq 2.5 \mathrm{~mm}$ in any region, $\mathrm{R} 2 \mathrm{~b}$, macroscopic residue $>2.5 \mathrm{~mm}$ in any region, NED, no evidence of disease, AWD, alive with disease, DOD, died of disease.

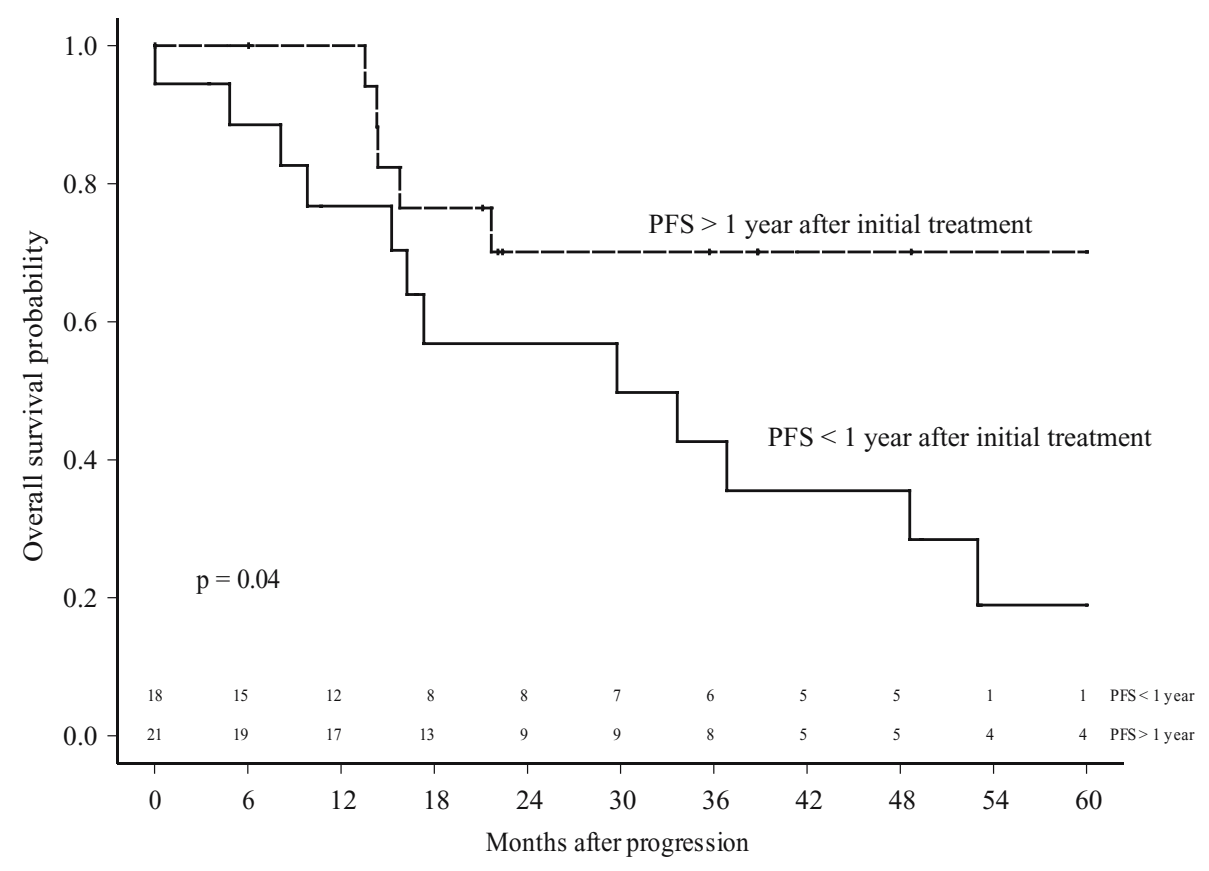

FIG. 2. Overall survival probability after progression related to the progression free survival after initial treatment in patients with progressive PMP after primary CRS and HIPEC. PMP, pseudomyxoma peritonei, CRS, cytoreductive surgery, HIPEC, hyperthermic intraperitoneal chemotherapy. showed no further progress during the 3 years after the diagnosis of progression. In one of these patients, with DPAM subtype, finally second CRS plus HIPEC was performed because of slowly diffuse progression. This patient was alive with disease at end of follow-up. Three-year OS probability of this treatment group was $66.0 \%$ (95\% CI 43.4-100\%). All patients that had died of disease had the PMCA-I subtype.

Six patients were treated for progressive disease with systemic chemotherapy. Three patients with initial DPAM subtype were treated this way as they had showed dedifferentiation into PMCA-I at progression.

\section{DISCUSSION}

PMP has been traditionally treated by limited CRS consisting of removal of the free mucus and resection of both the appendiceal primary and dominant metastatic sites such as the ovaries and omentum. In the 
short run this approach has been safe and successful in improving the patient's well being. Recurrence or progression is however inevitable and leads to further surgery that is usually less successful. Intervals get shorter and eventually all surgical options are exhausted or patients die as consequence of surgical complications. ${ }^{13}$ More aggressive surgery, which aims at complete removal of tumor in all affected areas, seems to prolong survival. Still in a recent series only $12 \%$ of patients remained free of disease at end of follow-up. ${ }^{14}$ The combination of complete CRS by the use of peritonectomy procedures and hyperthermic intraperitoneal chemotherapy (HIPEC) aims to further reduce the percentage of recurrence and progression. A number of series using this approach has now been published, showing indeed that longterm disease free and progression free survival can be improved. ${ }^{3-6,15,16}$ Still, even after this combined modality treatment progression was observed in our series in $41 \%$ of cases. In other published series recurrence or progression rates of $40-70 \%$ have been reported. ${ }^{6,7,15}$ As in other series, we observed that pathological subtype and result of initial cytoreduction were the dominant factors related to progression. . $^{3,10,17}$

The diagnosis of progressive disease is not always easy. After extensive surgery CT scans are usually difficult to interpret due to post-surgical changes. We found it useful to make a baseline CT scan 3 months after primary CRS plus HIPEC and compare further CT scans with that. Progressive disease was defined as a marked raise of serum tumor markers and/or progressive masses on CT scan.

A dominant site of progressive disease has been the sub hepatic region. This is the area in which it is technically very difficult to reach a complete cytoreduction. This strongly suggests that incomplete resection is the dominant factor causing progression. Apparently HIPEC is not effective when large tumor residue is left behind. This is in accordance with our understanding of the action of HIPEC, which is probably only reaching an effective drug/heat doses at a superficial level. Thus, progressive disease in one or two regions probably represents surgical failure, not HIPEC failure.

In contrast, $36 \%$ of progressive disease was spread more diffuse in the abdomen, including areas that were left macroscopically free of disease. These patients seem to represent the failure of HIPEC to sterilize microscopic disease. Although this did not reach statistical significance, PMP with more malignant features (PMCA-I/dedifferentiated DPAM) was overrepresented in this pattern of failure. It seems that these cases represent the pattern of failure caused by the biology of PMP itself.

Laparotomy scar, top of the vagina and bowel suture lines were incidentally observed to harbor progressive disease. In these cases incomplete exposure to the perfusion fluid was probably the cause of progression. In five patients progressive disease was found outside the abdominal cavity, especially in the thoracic cavity $(n=4)$. Opening of routes to extra peritoneal spaces during CRS is probably the cause of this way of dissemination. ${ }^{8,18}$ Since we leave the diaphragm open during perfusion after CRS in this area, so effectively including the involved pleural cavity in the perfusion, this has not been observed anymore. In one patient metastasis to the bones was observed. As PMP is a non-invasive neoplasm, this true metastatic disease is probably the result of dedifferentiation into PMCA.

The management of progressive PMP during this 10 -year study period has been influenced by our increasing experience. Consequently not always the same treatment choices have been made. This makes it difficult to draw firm conclusions out of this experience. The progression free survival after initial CRS and HIPEC is the only statistically significant factor predicting long-term survival after treatment for progressive disease. This seems closely related to tumor biology. In patients with PMCA-I and in patients presenting with dedifferentiated progressive disease the progression free survival tended to be short and the outcome of treatment of progressive disease poor. Based on our experience we now use the following algorithm.

Patients with early progressive disease (short PFS) diffusely spread throughout the abdomen, usually PMCA-I subtype, are treated with (palliative) systemic chemotherapy, as prognosis in these patients is already very poor. However, the effect of chemotherapy is questionable as all patients in our series treated this way died within 3 years.

Patients with early progressive disease limited to one or two regions are treated with simple resection. Such limited (residual) disease can easily be resected one more time. It seems obvious though, that patients with PMCA-I subtype have less chance of improved survival than DPAM patients as result of tumor biology.

Patients with late progressive disease (long PFS) diffusely spread throughout the abdomen or diffuse progression after simple resection, and in good general health, are treated with a second procedure of CRS and HIPEC. These patients might benefit from another curative attempt. 
Besides there is a group of patients with very indolent tumor behavior, with limited disease and hardly any progression over a long period of time, who are simply followed. In these cases it is probably worthwhile to observe before deciding to perform surgery with all its disadvantages.

Because of the inherent differences in prognosis in these different patient groups it is impossible to judge the relative effectiveness of these different approaches. The exception is the group that received systemic chemotherapy. We did not observe any objective response on $5 \mathrm{FU}$ based chemotherapy in these patients. It is questionable whether modern schedules including Capecitabin, Oxaliplatin and Bevacuzimab will do any better.

Notwithstanding the limitations of this study it is clear that management of progressive PMP after primary aggressive CRS and HIPEC is worthwhile and can result in long-term survival in a considerable percentage of patients. A second HIPEC is probably best restricted to patients with benign disease and progression free survival exceeding 1 year.

\section{REFERENCES}

1. Sugarbaker PH. Pseudomyxoma peritonei. A cancer whose biology is characterized by a redistribution phenomenon. Ann Surg 1994; 219:109-11.

2. Deraco M, Gronchi A, Mazzaferro V, et al. Feasibility of peritonectomy associated with intraperitoneal hyperthermic perfusion in patients with Pseudomyxoma peritonei. Tumori 2002; 88:370-5.

3. Elias D, Laurent S, Antoun S, Duvillard P, Ducreux M, Pocard M, Lasser P. [Pseudomyxoma peritonei treated with complete resection and immediate intraperitoneal chemotherapy]. Gastroenterol Clin Biol 2003; 27:407-12.

4. Sugarbaker PH. Cytoreductive surgery and peri-operative intraperitoneal chemotherapy as a curative approach to pseudomyxoma peritonei syndrome. Eur J Surg Oncol 2001; 27:239-43.

5. Guner Z, Schmidt U, Dahlke MH, Schlitt HJ, Klempnauer J, Piso P. Cytoreductive surgery and intraperitoneal chemother- apy for pseudomyxoma peritonei. Int $J$ Colorectal Dis 2005; 20:155-60.

6. Deraco M, Baratti D, Inglese MG, Allaria B, Andreola S, Gavazzi C, Kusamura S. Peritonectomy and intraperitoneal hyperthermic perfusion (IPHP): a strategy that has confirmed its efficacy in patients with pseudomyxoma peritonei. Ann Surg Oncol 2004; 11:393-8.

7. Bryant J, Clegg AJ, Sidhu MK, Brodin H, Royle P, Davidson P. Systematic review of the Sugarbaker procedure for pseudomyxoma peritonei. Br J Surg 2005; 92:153-8.

8. Zoetmulder FA, Sugarbaker PH. Patterns of failure following treatment of pseudomyxoma peritonei of appendiceal origin. Eur J Cancer 1996; 32A:1727-33.

9. Gough DB, Donohue JH, Schutt AJ, et al. Pseudomyxoma peritonei. Long-term patient survival with an aggressive regional approach. Ann Surg 1994; 219:112-9.

10. Sugarbaker PH, Fernandez-Trigo V, Shamsa F. Clinical determinants of treatment failure in patients with pseudomyxoma peritonei. Cancer Treat Res 1996; 81:121-32.

11. Verwaal VJ, van Tinteren H, Ruth SV, Zoetmulder FA. Toxicity of cytoreductive surgery and hyperthermic intra-peritoneal chemotherapy. J Surg Oncol 2004; 85:61-7.

12. Ronnett BM, Zahn CM, Kurman RJ, Kass ME, Sugarbaker $\mathrm{PH}$, Shmookler BM. Disseminated peritoneal adenomucinosis and peritoneal mucinous carcinomatosis. A clinicopathologic analysis of 109 cases with emphasis on distinguishing pathologic features, site of origin, prognosis, and relationship to “pseudomyxoma peritonei”. Am J Surg Pathol 1995; 19:1390 1408.

13. Lang H, Jahne J, Flemming P, Meyer HJ, Pichlmayr R. Pseudomyxoma peritonei of appendiceal origin-a report of seven cases and a review of published reports. Eur J Surg 1995; 161:355-60.

14. Miner TJ, Shia J, Jaques DP, Klimstra DS, Brennan MF, Coit DG. Long-term survival following treatment of pseudomyxoma peritonei: an analysis of surgical therapy. Ann Surg 2005; 241:300-8.

15. Moran BJ, Mukherjee A, Sexton R. Operability and early outcome in 100 consecutive laparotomies for peritoneal malignancy. Br J Surg 2005; 93(1):100-4.

16. Loungnarath R, Causeret S, Bossard N, et al. Cytoreductive surgery with intraperitoneal chemohyperthermia for the treatment of pseudomyxoma peritonei: a prospective study. Dis Colon Rectum 2005; 48:1372-79.

17. Yan H, Pestieau SR, Shmookler BM, Sugarbaker PH. Histopathologic analysis in 46 patients with pseudomyxoma peritonei syndrome: failure versus success with a second-look operation. Mod Pathol 2001; 14:164-71.

18. Smeenk RM, Bex A, Verwaal VJ, Horenblas S, Zoetmulder FA. Pseudomyxoma peritonei and the urinary tract: involvement and treatment related complications. J Surg Oncol 2006; 93:20-3. 